\title{
PREVALÊNCIA DE HIPOVITAMINOSE A EM PRÉ-ESCOLARES DE MUNICÍPIO DA ÁREA METROPOLITANA DE SÃO PAULO, BRASIL*
}

\author{
Maria José Roncada** \\ Donald Wilson** \\ Eliza Tieko Okani** \\ Seiko Amino**
}

\begin{abstract}
RONCADA, M. J. et al. Prevalência de hipovitaminose A em préescolares de município da Área Metropolitana de São Paulo, Brasil. Rev. Saúde públ., S. Paulo, 18: 218 - 24, 1984.

RESUMO: Foram realizadas dosagens de vitamina A e caroteno plasmáticos em 362 pré: escolares de 3 a 6 anos de idade, residentes na área urbana do Município de Cotia, situado a $34 \mathrm{~km}$ de Saro Paulo, SP (Brasil), usando-se amostra representativa. O método utilizado foi o de Carr-Price e os critérios de classificaçăo dos resultados foram propostos pelo "Interdepartmental Committee on Nutrition for National Defense" (ICNND). A prevalência de hipovitaminose A em nível bioquímico foi de $30,2 \%$.
\end{abstract}

UNITERMOS: Vitamina A, deficiência. Caroteno. Inquéritos nutricionais.

\section{INTRODUÇÃO}

A ingestão reduzida de alimentos ricos em vitamina $A$, por tempo prolongado, causa diminuição da reserva hepática desse nutriente, e consequiente abaixamento dos níveis circulantes, estabelecendo-se, assim, a hipovita. minose A. Esta moléstia carencial poderá levar a alterações oculares de diferentes graus de intensidade, culminando em cegueira, parcial ou total, que uma vez instalada $\dot{e}$ irreversivel.

No Brasil foi constatado, em nivel nacional, pela Fundação Instituto Brasileiro de Geografia e Estatística (FIBGE), por meio do Estudo Nacional de Despesa Familiar $(\mathrm{ENDEF})^{23,4,5}$, grande inadequação no consumo de vitamina $A$, pois os resultados, em quase todos os locais pesquisados, foram geralmente inferiores a $100 \%$ de adequação. Tais resultados concordam com aqueles relatados por Roncada e col. ${ }^{9}$, referindo-se a onze comunidades paulistas estudadas pelo Departamento de Nutrição da Faculdade de Saúde Pública da Universidade de São Paulo.

No que se refere às informações bioquímicas, não há dados a respeito do país como um todo. Por isso, especialistas reunidos pelo Instituto Nacional de Alimentação e Nutrição (INAN) ${ }^{6}$ concluiram que os dados existentes são em pequeno número e, geralmente, pouco consistentes para que se possa definir com segurança o problema da hipovitaminose A no Brasil.

Dado o interesse do INAN em obter subsídios para a implementação de um sistema diagnóstico e de vigilância nutricional em relação à hipovitaminose $\mathrm{A}$, bem como para programas de combate a essa carência, foi firmado um convênio entre essa entidade $e \mathrm{a}$ Universidade de São Paulo, através de sua Faculdade de Saúde Pública, para a execução do Projeto Hipovitaminose A, cujo primeiro objetivo, escopo deste trabalho, é o levan-

* Trabalho subvencionado pelo Convênio no 08/81, realizado entre o Instituto Nacional de Alimentação e Nutrição (INAN) e a Faculdade de Saúde Pública/USP.

** Do Departamento de Nutrição da Faculdade de Saúde Pública da USP - Av. Dr. Arnaldo, 715 01255 - São Paulo, SP - Brasil. 
RONCADA, M. J. et al. Prevalência de hipovitaminose A em pré-escolares de município da Área Metropolitana de São Paulo, Brasil. Rev. Saúde públ., S. Paulo, 18: 218-24, 1984.

tamento da prevalência de hipovitaminose A em pré-escolares expostos ao risco.

\section{METODOLOGIA}

\section{Universo}

0 universo deste trabalho foi a população de 3 a 6 anos de idade, residente na área urbana do Município de Cotia, São Paulo, um dos 37 municípios integrantes da Grande São Paulo.

O Município de Cotia situa-se a oeste da Ãrea Metropolitana de São Paulo, ocupando uma superfície de $417 \mathrm{~km}^{2}$. Sua sede locali. za-se a $34 \mathrm{~km}$ de São Paulo, possuindo clima temperado e altitude média de $750 \mathrm{~m}$.

0 censo de 1980 mostrou uma população de 62.569 habitantes, $51 \%$ do sexo mas culino e $49 \%$ do feminino, havendo predominância dos grupos etários mais jovens ( $37,3 \%$ com menos de 15 anos de idade).

\section{Amostragem}

Cadastramento da População - Foi realizado um cadastramento para o qual utilizou-se uma ficha especificamente elaborada para este fim. Inicialmente, foi feita a atualização da planta da cidade fornecida pela Prefeitura Municipal (Planta de 1978). Em seguida, visitaram-se todas as residências do perímetro urbano, sendo as informações necessárias para o preenchimento da ficha de cadastramento solicitadas a indivíduos adultos residentes na moradia visitada.

Este cadastramento permitiu separar as famílias residentes na área que contavam em sua composição com pré-escolares de 3 a 6 anos de idade, restringindo-se, assim, o universo. Preencheram tal requisito 1.083 familias, totalizando 1.426 crianças com a idade exigida.

Extração da Amostra - As famílias componentes deste universo restrito foram numeradas arbitrariamente, utilizando-se uma tabela de números ao acaso; sorteou-se um número de-familias de modo a ter uma amostra de 500 pré-escolares de 3 a 6 anos, representada pela soma dos pré-escolares de cada familia.

0 mesmo critério foi utilizado sempre que se tornou necessário repor uma familia perdida por recusa ou mudança de domicílio.

Após a extração da amostra, foi ela comparada ao universo, segundo os atributos sexo, idade e local de residência, revelandose representativa.

\section{Colheita de sangue e processamento das amostras}

Foram colhidos de cada criança $5 \mathrm{ml}$ de sangue por venopunção, usando material descartável (equipamento Vacutainer ${ }^{\circledR}$ ) contendo etilenodiamintetracetato dissódico-EDTA- como anti-coagulante.

As amostras eram cuidadosamente misturadas por inversão e colocadas em caixa de isopor fechada e resfriada, onde permaneciam até sua chegada ao laboratório do Departamento de Nutrição da Faculdade de Saúde Pública da Universidade de São Paulo, no qual foram realizadas as análises.

O processamento das amostras de sangue no laboratório dava-se até 3 horas após a colheita, quando então os tubos eram homogeneizados para a retirada das alíquotas que serviriam à micro-determinação de hemoglobina*; em seguida, o plasma era separado por centrifugação a 3.000 r.p.m. por $10 \mathrm{~min}$, colocado em tubos plásticos de cor âmbar, submetido a tratamento com nitrogênio $e$ congelado a $\cdot 20^{\circ} \mathrm{C}$. As análises de vitamina $\mathrm{A}$ e caroteno foram realizadas em prazo que variou entre o mesmo dia da colheita das amostras até um máximo de 10 dias.

A condição "sine qua non" para a realização da colheita de sangue foi a anuência dos responsáveis pelos pré-escolares.

\section{Método bioquímico}

0 método adotado para as dosagens de vitamina $\mathrm{A}$ e caroteno plasmáticos foi o de

\footnotetext{
* Dados Inéditos.
} 
RONCADA, M. J. et al. Prevalência de hipovitaminose A em pré-escolares de município da Área Metropolitana de São Paulo, Brasil. Rev. Saúde públ., S. Paulo, 18: 218-24, 1984.

Carr-Price ${ }^{1}$, conforme recomendação do International Vitamin A Consultative Group (IVACG) $^{10}$ para trabalhos de campo e também por ser preferivel, segundo Strohecker e Henning ", na determinação de vitamina A em compostos nos quais sua concentração é baixa, como no caso do leite e do sangue. Utilizou-se espectrofotômetro Coleman 295.

Houve a preocupação de proteger as amostras contra a ação da luz e da oxidação em todas as fases (colheita, transporte, manuseio, armazenamento e análise), sendo descartadas as amostras hemolisadas. $O$ controle de qualidade foi realizado com amostras. controle igualmente conservadas a $-20^{\circ} \mathrm{C}$.

\section{Critérios para interpretação dos resultados}

Utilizaramse os critérios propostos pelo Interdepartmental Committee on Nutrition for National Defense (ICNND) ${ }^{7}$ :

a) Para vitamina A plasmática:

Deficiente (D) - $<10 \mu \mathrm{g} / 100 \mathrm{ml}$

Baixo (B) $\cdot 10 \cdot 19,9 \mu \mathrm{g} / 100 \mathrm{ml}$

Aceitável (A) $-20-49,9 \mu \mathrm{g} / 100 \mathrm{ml}$

Alto $\quad(\mathrm{H})-\geqslant 50 \mu \mathrm{g} / 100 \mathrm{ml}$

b) Para caroteno plasmático:

Deficiente (D) $-<20 \mu \mathrm{g} / 100 \mathrm{ml}$
Baixo (B) - $20-39,9 \mu \mathrm{g} / 100 \mathrm{ml}$

Aceitável (A) $\cdot 40 \cdot 99,9 \mu \mathrm{g} / 100 \mathrm{ml}$

Alto $\quad(\mathrm{H}) \cdot \geqslant 100 \mu \mathrm{g} / 100 \mathrm{ml}$

Também, segundo o ICNND, existe um problema de Saúde Pública com relação à vitamina A quando $5 \%$ ou mais da amostra apresentam um valor plasmático de vitamina A menor do que $10 \mu \mathrm{g} / 100 \mathrm{ml}$ ou $15 \%$ abaixo de $20 \mu \mathrm{g} / 100 \mathrm{ml}$. Deve-se enfatizar que os dados bioquímicos tomados isoladamente sáo indicativos apenas de carência de vitamina $A$, não expressando um problema de xeroftalmia.

\section{RESULTADOS E DISCUSSÃO}

Como costuma ocorrer em trabalhos desta natureza, não se conseguiu colher sangue de um certo número de crianças, ou porque os responsáveis não permitiram a colheita ou por dificuldades técnicas. No presente trabalho colheu-se sangue de apenas 362 préescolares.

Examinado-se a Tabela 1, pode-se observar que os resultados médios, em todas as categorias de idade e nos dois sexos, são superiores a $20 \mu \mathrm{g} / 100 \mathrm{ml}$, considerado valor limite de normalidade ${ }^{7}$. Não houve variaçб̃es significantes segundo idade ou sexo.

TA B E L A 1

Média e desvio-padráo de resultados das dosagens de vitamina A plasmática (em $\mu \mathrm{g} / 100 \mathrm{ml}$ ), segundo idade e sexo, dos pré-escolares estudados. Cotia, São Paulo, 1982/83.

\begin{tabular}{|c|c|c|c|c|c|c|}
\hline \multirow{3}{*}{$\begin{array}{l}\text { Idade } \\
\text { (anos) }\end{array}$} & \multicolumn{6}{|c|}{ Sexo } \\
\hline & \multicolumn{3}{|c|}{ Feminino } & \multicolumn{3}{|c|}{ Masculino } \\
\hline & No & Média & $\begin{array}{l}\text { Desvio } \\
\text { Padráo }\end{array}$ & NQ & Média & $\begin{array}{l}\text { Desvio } \\
\text { Padrão }\end{array}$ \\
\hline $\begin{array}{l}3 \\
4 \\
5 \\
6\end{array}$ & $\begin{array}{l}48 \\
42 \\
44 \\
40\end{array}$ & $\begin{array}{l}23,6 \\
23,7 \\
25,6 \\
23,3\end{array}$ & $\begin{array}{l}7,2 \\
8,1 \\
9,4 \\
8,5\end{array}$ & $\begin{array}{l}46 \\
58 \\
43 \\
40\end{array}$ & $\begin{array}{l}24,6 \\
24,2 \\
24,7 \\
24,9\end{array}$ & $\begin{array}{l}7,2 \\
7,3 \\
7,5 \\
5,9\end{array}$ \\
\hline
\end{tabular}


RONCADA, M. J. et al. Prevalência de hipovitaminose A em pré-escolares de município da Área Metropolitana de São Paulo, Brasil. Rev. Saúde públ., S. Paulo, 18: 218-24, 1984.

TA B E L A 2

Distribuiçăo dos resultados das dosagens de vitamina A plasmática, segundo classificação do ICNND, idade e sexo dos pré-escolares estudados. Cotia, São Paulo, $1982 / 83$.

\begin{tabular}{|c|c|c|c|c|c|c|c|c|c|c|}
\hline \multirow{3}{*}{$\begin{array}{l}\text { Idade } \\
\text { (anos) }\end{array}$} & \multirow{3}{*}{ Sexo } & \multirow{3}{*}{$\begin{array}{l}\text { Nọ de } \\
\text { Crianças }\end{array}$} & \multicolumn{8}{|c|}{ Classificação dos Resultados (ICNND) } \\
\hline & & & \multicolumn{2}{|c|}{ D } & \multicolumn{2}{|c|}{ B } & \multicolumn{2}{|c|}{ A } & \multicolumn{2}{|c|}{ H } \\
\hline & & & Nọ & $\%$ & Nọ & $\%$ & No & $\%$ & Nọ & $\%$ \\
\hline 3 & $\stackrel{\mathrm{M}}{\mathrm{F}}$ & $\begin{array}{l}46 \\
48\end{array}$ & $\begin{array}{l}2 \\
1\end{array}$ & $\begin{array}{l}4,3 \\
2,1\end{array}$ & $\begin{array}{l}12 \\
15\end{array}$ & $\begin{array}{l}26,1 \\
31,2\end{array}$ & $\begin{array}{l}32 \\
32\end{array}$ & $\begin{array}{l}69,6 \\
66,7\end{array}$ & $\begin{array}{l}0 \\
0\end{array}$ & $\begin{array}{l}0 \\
0\end{array}$ \\
\hline 4 & $\begin{array}{l}\mathrm{M} \\
\mathrm{F}\end{array}$ & $\begin{array}{l}58 \\
42\end{array}$ & $\begin{array}{l}2 \\
2\end{array}$ & $\begin{array}{l}3,4 \\
4,8\end{array}$ & $\begin{array}{l}15 \\
14\end{array}$ & $\begin{array}{l}25,9 \\
33,3\end{array}$ & $\begin{array}{l}41 \\
26\end{array}$ & $\begin{array}{l}70,7 \\
61,9\end{array}$ & $\begin{array}{l}0 \\
0\end{array}$ & $\begin{array}{l}0 \\
0\end{array}$ \\
\hline 5 & $\begin{array}{l}\mathrm{M} \\
\mathrm{F}\end{array}$ & $\begin{array}{l}43 \\
44\end{array}$ & $\begin{array}{l}1 \\
2\end{array}$ & $\begin{array}{l}2,3 \\
4,5\end{array}$ & $\begin{array}{l}10 \\
12\end{array}$ & $\begin{array}{l}23,3 \\
27,3\end{array}$ & $\begin{array}{l}32 \\
30\end{array}$ & $\begin{array}{l}74,4 \\
68,2\end{array}$ & $\begin{array}{l}0 \\
0\end{array}$ & $\begin{array}{l}0 \\
0\end{array}$ \\
\hline 6 & $\begin{array}{l}\mathrm{M} \\
\mathrm{F}\end{array}$ & $\begin{array}{l}40 \\
40\end{array}$ & $\begin{array}{l}0 \\
3\end{array}$ & $\begin{array}{l}0 \\
7,5\end{array}$ & $\begin{array}{r}6 \\
12\end{array}$ & $\begin{array}{l}15,0 \\
30,0\end{array}$ & $\begin{array}{l}34 \\
25\end{array}$ & $\begin{array}{l}85,0 \\
62,5\end{array}$ & $\begin{array}{l}0 \\
0\end{array}$ & $\begin{array}{l}0 \\
0\end{array}$ \\
\hline $\begin{array}{l}\text { Todas as } \\
\text { e ambos }\end{array}$ & $\begin{array}{l}\text { es } \\
\text { xos }\end{array}$ & 361 & 13 & 3,6 & 96 & 26,6 & 252 & 69,8 & 0 & 0 \\
\hline
\end{tabular}

Levando-se em consideração, por outro lado, a classificação de resultados proposta pelo ICNND, observa-se uma alteração nesse quadro. Assim, vê-se na Tabela 2 que 13 crianças $(3,6 \%)$ apresentaram valores de vitamina A plasmática $<10 \mu \mathrm{g} / 100 \mathrm{ml}$ e 96 delas $(26,6 \%)$ valores entre 10 e $19,9 \mu \mathrm{g} / 100 \mathrm{ml}$. Portanto, segundo o ICNND, 109 crianças $(30,2 \%)$ têm resultados abaixo do limite mínimo normal, ou seja, quase um terço das crianças examinadas bioquimicamente. Além disso, chama a atenção o fato de que nenhuma das crianças apresentou um valor considerado "Alto", por essa classificação. Também não houve variações significantes quanto a sexo ou idade.

Em Lomba do Tarumã (Viamão, RS), Wilson e Nery ${ }^{12}$ encontraram $43,9 \%$ de resultados de dosagens bioquímicas de vitamina A em pré-escolares abaixo de $20 \mu \mathrm{g} / 100 \mathrm{ml}$, sendo $24,2 \%$ menores que $10 \mu \mathrm{g} / 100 \mathrm{ml}$. Roncada e col. ${ }^{8}$, estudando crianças de 2 a 7 anos de idade, filhos de migrantes nacionais em trânsito pela Capital de São Paulo, encontraram $51,4 \%$ de valores plasmáticos de vitamina $\mathrm{A}$ abaixo do normal, dos quais $13,9 \%$ classificados como "Deficiente". Wilson e col. ${ }^{13}$, examinando pré-escolares internados em uma instituição para menores, também na Capital, verificaram que $73,9 \%$ dos examinados apresentavam valores plásmáticos de vitamina $\mathrm{A}$ abaixo de $20 \mu \mathrm{g} / 100$ $\mathrm{ml}$, compreendendo $39,4 \%$ na categoria "Deficiente".

Lembrando o critério estabelecido pelo ICNND para caracterizar a hipovitaminose A como problema de Saúde Pública, verifica-se existir tal problema na localidade em pauta, embora não apresentando a mesma magnitude desses outros grupos estudados pelo Departamento de Nutrição. $O$ fato deve, porém, ser encarado com muita atenção, dada a informação que fornece a programas de combate à desnutrição e a sistemas de vigilância nutricional.

Quanto ao caroteno plasmático, que exprime a ingestão recente de alimentos ricos em pró-vitaminas $A$, os resultados estão expressos às Tabelas 3 e 4 .

Os valores médios das dosagens de carote- 
RONCADA, M. J. et à. Prevalência de hipovitaminose A em pré-escolares de município da Área Metropolitana de São Paulo, Brasil. Rev. Saŕde públ., S. Paulo, 18: 218-24, 1984.

T A B E L A 3

Média e desvio-padrão de resultados das dosagens de caroteno plasmático (em $\mu \mathrm{g} / 100 \mathrm{ml}$ ), segundo idade e sexo, dos pré escolares estudados. Cotia, Sáo Paulo, 1982/83.

\begin{tabular}{|c|c|c|c|c|c|c|}
\hline \multirow{3}{*}{$\begin{array}{l}\text { Idade } \\
\text { (anos) }\end{array}$} & \multicolumn{6}{|c|}{ Sexo } \\
\hline & \multicolumn{3}{|c|}{ Feminino } & \multicolumn{3}{|c|}{ Masculino } \\
\hline & No & Média & $\begin{array}{l}\text { Desvio } \\
\text { Padrão }\end{array}$ & Nọ & Média & $\begin{array}{l}\text { Desvio } \\
\text { Padrão }\end{array}$ \\
\hline $\begin{array}{l}3 \\
4 \\
5 \\
6\end{array}$ & $\begin{array}{l}48 \\
42 \\
44 \\
40\end{array}$ & $\begin{array}{l}72,0 \\
83,8 \\
82,0 \\
68,3\end{array}$ & $\begin{array}{l}27,1 \\
35,7 \\
36,9 \\
22,3\end{array}$ & $\begin{array}{l}46 \\
58 \\
44 \\
40\end{array}$ & $\begin{array}{l}61,6 \\
64,3 \\
68,9 \\
75,4\end{array}$ & $\begin{array}{l}25,0 \\
26,6 \\
29,1 \\
29,6\end{array}$ \\
\hline
\end{tabular}

TAB E L A 4

Distribuição dos resultados das dosagens de caroteno plasmático, segundo classificação do ICNND, idade e sexo dos pré-escolares estudados. Cotia, São Paulo, 1982/83.

\begin{tabular}{|c|c|c|c|c|c|c|c|c|c|c|}
\hline \multirow{3}{*}{$\begin{array}{l}\text { Idade } \\
\text { (anos) }\end{array}$} & \multirow{3}{*}{ Sexo } & \multirow{3}{*}{$\begin{array}{r}\text { No de } \\
\text { Crianças }\end{array}$} & \multicolumn{8}{|c|}{ Classificação dos Resultados (ICNND) } \\
\hline & & & \multicolumn{2}{|c|}{ D } & \multicolumn{2}{|c|}{ B } & \multicolumn{2}{|c|}{$\mathbf{A}$} & \multicolumn{2}{|c|}{$\mathbf{H}$} \\
\hline & & & NQ & $\%$ & No & $\%$ & No & $\%$ & No & $\%$ \\
\hline 3 & $\begin{array}{l}\mathbf{M} \\
\mathbf{F}\end{array}$ & $\begin{array}{l}46 \\
48\end{array}$ & $\begin{array}{l}2 \\
0\end{array}$ & $\begin{array}{c}4,3 \\
0\end{array}$ & $\begin{array}{l}6 \\
5\end{array}$ & $\begin{array}{l}13,1 \\
10,4\end{array}$ & $\begin{array}{l}34 \\
35\end{array}$ & $\begin{array}{l}73,9 \\
72,9\end{array}$ & $\begin{array}{l}4 \\
8\end{array}$ & $\begin{array}{r}8,7 \\
16,7\end{array}$ \\
\hline 4 & $\begin{array}{c}\mathrm{M} \\
\mathrm{F}\end{array}$ & $\begin{array}{l}58 \\
42\end{array}$ & $\begin{array}{l}1 \\
0\end{array}$ & $\begin{array}{c}1,7 \\
0\end{array}$ & $\begin{array}{l}8 \\
4\end{array}$ & $\begin{array}{r}13,8 \\
9,5\end{array}$ & $\begin{array}{l}42 \\
26\end{array}$ & $\begin{array}{l}72,4 \\
61,9\end{array}$ & $\begin{array}{r}7 \\
12\end{array}$ & $\begin{array}{l}12,1 \\
28,6\end{array}$ \\
\hline 5 & $\begin{array}{l}\mathbf{M} \\
\mathbf{F}\end{array}$ & $\begin{array}{l}44 \\
44\end{array}$ & $\begin{array}{l}1 \\
1\end{array}$ & $\begin{array}{l}2,3 \\
2,3\end{array}$ & $\begin{array}{l}4 \\
2\end{array}$ & $\begin{array}{l}9,1 \\
4,5\end{array}$ & $\begin{array}{l}33 \\
29\end{array}$ & $\begin{array}{l}75,0 \\
65,9\end{array}$ & $\begin{array}{r}6 \\
12\end{array}$ & $\begin{array}{l}13,6 \\
27,3\end{array}$ \\
\hline 6 & $\begin{array}{l}\mathbf{M} \\
\mathbf{F}\end{array}$ & $\begin{array}{l}40 \\
40\end{array}$ & $\begin{array}{l}0 \\
1\end{array}$ & $\begin{array}{c}0 \\
2,5\end{array}$ & $\begin{array}{l}4 \\
3\end{array}$ & $\begin{array}{r}10,0 \\
7,5\end{array}$ & $\begin{array}{l}30 \\
32\end{array}$ & $\begin{array}{l}75,0 \\
80,0\end{array}$ & $\begin{array}{l}6 \\
4\end{array}$ & $\begin{array}{l}15,0 \\
10,0\end{array}$ \\
\hline $\begin{array}{l}\text { Todas as } \\
\text { ambos }\end{array}$ & $\begin{array}{l}\text { es } \\
\text { xos }\end{array}$ & 362 & 6 & 1,7 & 36 & 9,9 & 261 & 72,1 & 59 & 16,3 \\
\hline
\end{tabular}

no plasmático revelaram-se acima do nível considerado normal $(40 \mu \mathrm{g} / 100 \mathrm{ml})$. Entretanto, pode-se notar que cerca de $11 \%$ das crianças apresentaram resultados que, pelo
ICNND, estão abaixo de "Aceitável", o que revela uma ingestão baixa de fontes de caro. tenóides, no mínimo por ocasião próxima à colheita de sangue. 
RONCADA, M. J. et al. Prevalência de hipovitaminose A em pré-escolares de município da Área Metropolitana de São Paulo, Brasil. Rev. Saúde públ., S. Paulo, 18:218-24, 1984.

\section{CONCLUSÕES}

1. A prevalência de hipovitaminose $\mathrm{A}$ em nível bioquímico nos pré-escolares de 3 a 6 anos de idade, na localidade de Cotia, é de $30,2 \%$.

2. Segundo o ICNND, a hipovitaminose A nesta localidade é problema de Saúde Pú. blica $(30,2 \%$ abaixo de $20 \mu \mathrm{g} / 100 \mathrm{ml})$, embora a proporção de valores na categoria

\section{"Deficiente" esteja aquém de $5 \%$.}

3. Em magnitude, o problema é inferior aos encontrados em outras pesquisas realizadas pelo Departamento de Nutrição da Faculdade de Saúde Pública da Universidade de São Paulo.

4. O caroteno plasmático apresenta proporção de $11,6 \%$ de valores considerados inferiores ao limite de normalidade.

RONCADA, M. J. et al. [Prevalence of hypovitaminosis A in preschool children in a town of the Metropolitan Area of S. Paulo, Brazil]. Rev. Saúde públ., S. Paulo, 18: $218 \cdot 24,1984$.

ABSTRACT: Three-hundred and sixty-two preschool children between the ages of three and six years were examined for plasma levels of vitamin $A$ and carotene. All were residents of the urban area of the city of Cotia, thirty-four $\mathrm{km}$ from S. Paulo, Brazil. A representative sample was taken for the purpose. The Carr-Price laboratory method was used as were the Interdepartmental Committee on Nutrition for National Defense (ICNND) criteria for classifying results. Prevalence of vitamin A deficiency at biochemical level was 30.2\%.

UNITERMS:Vitamin A, deficiency. Carotene. Nutritional surveys.

\section{REFERÊNCIAS BIBLIOGRÁFICAS}

1. CARR, F. H. \&PRICE, E. A. Colour reactions attributed to vitamin A. Biochem. J., 20 497-501, 1926.

2. FUNDAÇÃO IBGE. Consumo alimentar; antropometria; Regiao I - Estado do Rio de Janeiro; Regido III - Parand́, Santa Catarina e Rio Grande do Sul. Rio de Janeiro, 1978. (Estudo Nacional da Despesa Familiar, v:1:dados preliminares, t.1).

3. FUNDAÇÃO IBGE. Consumo alimentar; antropometria; Regido II - São Paulo; Regiāo IV - Minas Gerais e Espírito Santo. Rio de Janeiro, 1977. (Estudo Nacional da Despesa Familiar, v.1:dados preliminares, t.1).

4. FUNDACุÃO IBGE. Consumo alimentar; antrópometria; Região $V$ - Maranhão, Piaui, Ceard, Rio Grande do Norte, Paraiba, Permambuco, Alagoas, Sergipe e Bahia. Rio de Janeiro, 1977. (Estudo Nacional da Despesa Familiar, v.1: dados preliminares, t.1).
5. FUNDAC ĀO IBGE. Consumo alimentar; antropometria; Região VI - Distrito Federal; Região VII - Rondônia, Acre, Amazonas, Roraima, Pard, Amapd, Goids e Mato Grosso. Rio de Janeiro, 1978. (Estudo Nacional da Despesa Familiar, v.1: dados preliminares, t.1).

6. INSTITUTO NACIONAL DE ALIMENTAÇÃO E NUTRIÇÃO. Hipovitaminose $A$ no Brasil. Brasília, 1977.

7. INTERDEPARTMENTAL COMMITTEE ON NUTRITION FOR NATIONAL DEFENSE. Manual for nutrition surveys. $2^{\text {nd }}$ ed. Washington, D. C., Government Printing Office, 1963.

8. RONCADA, M. J.; WILSON, D.; LUI NETTO, A.; BERRETTA NETTO, O.; KALIL, C. A.; NUNES, M. F. \& OKANI, E.T. Hipovitaminose $A$ em filhos de migrantes nacionais em trânsito pela Capital do Estado de São Paulo, Brasil. Estudo clínicobioquímico. Rev. Saúde públ., S. Paulo, 12: 345-50, 1978. 
RONCADA, M. J. et al. Prevalência de hipovitaminose A em pré-escolares de município da Área Metropolitana de São Paulo, Brasil. Rev. Saŕde públ., S. Paulo, 18:218-24, 1984.

9. RONCADA, M. J.; WILSON, D.; MAZZILLI, R. N. \&GANDRA, Y. R. Hipovitaminose A em comunidades do Estado de São Paulo, Brasil. Rev. Saúde públ., S. Paulo, 15: 338 - 49, 1981.

10. SOMMER, A.; MCLAREN, D. S. \&OLSON, J. A. Assessment of vitamin A status. IVACG Task Force on Assessment. In: Guidelines for the eradication of vitamin $A$ deficiency and xerophthalmia; a report of the International Vitamin A Consultative Group (IVACG). New York, The Nutrition Foundation, 1976. p.I-1-I$-7$.
11.STROHECKER, R. \&HENNING, H. M. Analisis de vitaminas: métodos comprobados. Madrid, Ed. Paz Montalvo, 1967.

12. WILSON, D. \& NERY, M. E. S. Hypovitaminosis $A$ in Rio Grande do Sul, Brazil. Preliminary study. Int. J. Vit. Nutr. Res. (suppl. 24): 35.44, 1983.

13.WILSON, D.; RONCADA, M. J.; LUI NETTO, A. \& BERRETTA NETTO, O. Hipovita. minose $A$ em pré-escolares internados em uma instituição na Capital do Estado de São Paulo, Brasil. Rev. Saúde públ., S. Paulo, 15: 395-400, 1981.

Recebido para publicação em 11/01/1984 Aprovado para publicaçäo em 26/03/1984 